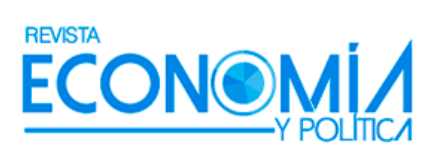

Revista Economía y Política

ISSN: $1390-7921$

revista.economiaypolitica@ucuenca.edu.ec

Universidad de Cuenca

Ecuador

\title{
Elasticidad-renta del comercio bilateral mediante el Modelo Gravitacional. Caso Ecuador
}

Tonon Ordóñez, Luis B.; Pinos Luzuriaga, Luis G.; Albornoz Flores, Ana C.; García Flores, Paulo E. Elasticidad-renta del comercio bilateral mediante el Modelo Gravitacional. Caso Ecuador

Revista Economía y Política, vol. XV, núm. 30, 2019

Universidad de Cuenca, Ecuador

DOI: https://doi.org/10.25097/rep.n30.2019.06

(C) Universidad de Cuenca 2019

Esta obra está bajo una Licencia Creative Commons Atribución-NoComercial-Compartirlgual 4.0 Internacional 
Artículos

\section{Elasticidad-renta del comercio bilateral mediante el Modelo Gravitacional. Caso Ecuador}

Income elasticity of bilateral trade through the Gravitational Model. Case Ecuador

Luis B. Tonon Ordónez

DOI: https://doi.org/10.25097/rep.n30.2019.06

Universidad del Azuay, Ecuador

ltonon@uazuay.edu.ec

iD http://orcid.org/0000-0003-2360-9911

Recepción: 16 Mayo 2019

Luis G. Pinos Luzuriaga

Aprobación: 01 Julio 2019

Universidad del Azuay, Ecuador

lpinos@uazuay.edu.ec

(iD http://orcid.org/0000-0002-3894-8652

Ana C. Albornoz Flores

Universidad del Azuay, Ecuador

anacalbornoz@es.uazuay.edu.ec

iD http://orcid.org/0000-0002-7512-3684

\section{Paulo E. García Flores}

Universidad del Azuay, Ecuador

paulogarciaflores@es.uazuay.edu.ec

iD http://orcid.org/0000-0003-0130-0112

Recepción: 16 Mayo 2019

Aprobación: 01 Julio 2019

\section{Resumen:}

Este trabajo determina la importancia estadística del Producto Interno Bruto en el flujo comercial del Ecuador con 19 socios comerciales del continente americano y obtiene la elasticidad-renta de los flujos comerciales bilaterales, con base en el modelo gravitacional de comercio exterior en lo referente al tamaño (masa) de las economías con las que comercian y la distancia que lo separan. Para este trabajo se estructuraron bases de datos con el PIB nominal de cada país y los flujos comerciales expresados en valores FOB, en dólares estadounidenses, y las distancias en kilómetros entre las capitales. Para el cálculo de las elasticidades se aplicó un modelo de Datos de Panel con efectos aleatorios para la obtención de los respectivos coeficientes.

Palabras clave: Modelo gravitacional, comercio bilateral, Ecuador, elasticidad de la renta, Producto Interno Bruto, Datos de Panel de efectos aleatorios.

\section{ABstract:}

This study determines the statistical importance of the Gross Domestic Product in the trade flow of Ecuador with 19 trading partners of the American continent and obtains the income elasticity of bilateral trade flows based on the gravity model of foreign trade, in terms of the size (mass) of the economies they trade with and the distance between them. For this work, databases were structured with the nominal GDP of each country and the trade flows expressed in FOB values, in U.S. dollars, and the distances in kilometers between the capitals. For the calculation of elasticities, a Panel Data model with random effects was applied to obtain the respective coefficients.

KEYWORDS: Gravity model, bilateral trade, Ecuador, income elasticity, Gross Domestic Product, Random Effects Panel Data. 


\section{INTRODUCCIÓN}

El volumen de comercio bilateral o flujo comercial está compuesto por la sumatoria de las importaciones y exportaciones, pero ¿qué determina en primera instancia este volumen de comercio? ¿Cuáles son sus variables básicas? Las diferentes teorías sostienen que estos flujos dependen, entre otras, de variables como el Producto Interno Bruto (PIB) de los países que comercian, el tipo de cambio real, los precios de los productos, los costos de transporte, aranceles, etc.

En base a lo antes mencionado, la hipótesis de esta investigación supone que el flujo comercial bilateral entre Ecuador y cada país de América, depende del PIB de los países seleccionados que forman parte de esta relación de comercio bilateral, es decir, depende del tamaño de las economías ¿Qué tan sensibles son los flujos de comercio a cambios en el PIB? Esto se responde mediante la obtención del coeficiente de elasticidad-renta, objetivo de esta investigación.

Por tanto, en este trabajo se estudia la elasticidad de los flujos comerciales del Ecuador con 19 países del continente americano, pues aproximadamente el 70\% de los flujos comerciales se dan con los países del mismo continente, estando en este, tres de sus principales socios comerciales: Estados Unidos de América, aproximadamente con $32,14 \%$ en promedio; Colombia con $7,71 \%$ y Perú con 5,48\%. Esto significa que alrededor del $45 \%$ del comercio de Ecuador se da con estos 3 estos países.

La sensibilidad de los flujos comerciales bilaterales debe ser tomada en cuenta como un elemento de juicio en las decisiones de la política comercial ecuatoriana y por lo mismo, los resultados plasmados en este estudio pueden ser preocupantes para los gestores de las políticas públicas. Hallazgos como una elasticidad renta mayor en las importaciones que en las exportaciones, implica la existencia de dificultades y lastres al desarrollo del país. La explicación de esto puede encontrarse en que los productos ecuatorianos, al parecer, no son atractivos ni para sus ciudadanos, ni para el extranjero cuando aumenta su PIB, por las características propias de la producción ecuatoriana, con muchas materias primas y productos de un bajo nivel de manufactura; lo que a su vez genera una disminución de la importancia de las distancias en las importaciones al verse que, con el crecimiento, los ecuatorianos buscarán productos que no se encuentran en el país y son difícilmente sustituibles.

Por lo antes mencionado, se debe prestar especial atención a las políticas a desarrollar con ciertos países del continente, como Perú. Aumentar las barreras o aumentar el número de oficinas comerciales depende del resultado de las elasticidades que tenga el Ecuador con los diferentes países. De esta manera, tomando en cuenta los datos, se pueden generar políticas que ayuden al desarrollo de la economía ecuatoriana, a la vez que se evitan posibles problemas futuros.

\section{REVISIÓN DE LITERATURA}

El Producto Interno Bruto es uno de los indicadores más utilizados en los estudios económicos, pues representa la producción de un país en un periodo determinado y a su vez, sería un proxy del tamaño de su economía y por ende, del ingreso nacional. Desde el punto de vista de las relaciones comerciales internacionales el tamaño de las economías de los países que negocian es uno de los determinantes de los flujos comerciales. La teoría toma en cuenta que las exportaciones de un país tienen, como una de sus variables explicativas, a la renta del país comprador, mientras que, las importaciones, a la renta nacional.

Para Montenegro, Pereira y Soloaga (2011), la importancia del análisis del PIB radica en que el crecimiento de este indicador en los países exportadores muestra su capacidad de oferta de bienes, y a la inversa, en los países importadores, muestra su capacidad de demanda de bien. Argumento complementado por lo dicho por De Benedictis y Taglioni (2013) sobre que "las grandes economías ofrecen bienes que son relativamente competitivos o abundantes en variedad, o ambos” por lo que se vuelven más atractivas.

Krugman, Obstfeld y Melitz (2012) afirman que: 
Las economías grandes tienden a gastar mucho en importaciones porque tienen mucha renta y que tienden a atraer grandes proporciones del gasto de otros países, porque producen una amplia gama de productos. Así que el comercio entre dos economías cualesquiera es mayor cuanto más grande sea cualquiera de las economías implicadas (p. 14).

Para Daniels, Radebaugh y Sullivan (2013) las diferencias entre países, en lo referente a su población, territorio, o acceso a recursos, generan diferencias en el comercio y afirman que "debido a que los países pequeños tienen menos acceso a recursos nacionales variados y a grandes mercados, generalmente son más dependientes del comercio internacional que los países grandes" (p. 19).

Para Yotov, Larch, Monteiro y Piermartini (2016) el término tamaño ya lleva una información muy útil acerca de la relación entre el tamaño del país y de los flujos comerciales bilaterales, a saber: los grandes productores exportan más a todos los destinos; los grandes o ricos mercados importan más de todas las fuentes; y los flujos comerciales entre los países serán mayores cuanto más similar, en tamaño, sean los socios comerciales.

Lo expuesto anteriormente pone en el tapete la pregunta ¿cuánto influyen los cambios en el PIB de los socios comerciales sobre sus flujos comerciales? ¿Es posible medir la sensibilidad del comercio exterior debida a cambios en el PIB de los países? La respuesta es afirmativa.

En economía, la medida de sensibilidad se denomina elasticidad. Su concepción moderna fue definida por primera vez por Alfred Marsall (1890). Al aplicar este concepto al tema tratado, se podría afirmar que la elasticidad es la medida de la sensibilidad del flujo comercial bilateral debida a cambios en cualquiera de sus variables explicativas, céteris páribus.

Zack y Dalle (2015) afirman que "Las elasticidades del comercio exterior miden el porcentaje de variación de las cantidades exportadas e importadas ante un cambio porcentual de otras variables, como pueden ser el ingreso o los precios relativos". Se ha analizado la elasticidad del comercio exterior desde mediados del siglo pasado, se podría decir que uno de los pioneros en estudiar el tema fue Orcutt (1950) quien realizó un análisis sobre la elasticidad precio en el comercio exterior. Harberger (1957) afirma que los primeros estudios sobre el tema fueron realizados por el Fondo Monetario Internacional mediante las estimaciones de Polak, donde se intentó explicar el efecto en las importaciones causados por los cambios porcentuales en el ingreso real, estudio realizado tomando en cuenta ocho países, principalmente mediante la aplicación de elasticidades del ingreso. Desde entonces se ha medido en función del PIB de los socios comerciales, del tipo de cambio real, de los precios relativos, tasas de interés, costos comerciales, etc.

En distintas investigaciones se han utilizado, para el cálculo de la elasticidad series de tiempo, técnicas de cointegración y modelos de corrección de errores, y en muchos casos las estimaciones de elasticidades de importaciones y exportaciones se han calculado por separado, esto sirve para evaluar el impacto del crecimiento económico en el saldo de la balanza comercial y por ende, en las políticas comerciales.

Los estudios de elasticidad de los flujos comerciales se han realizado tanto para países desarrollados, como para países en desarrollo.

Dutt Amitava (2002), por medio del método de Mínimos Cuadrados Ordinarios, analiza la elasticidad ingreso de la demanda de importaciones en el comercio entre países ricos y pobres, o comercio NorteSur. Afirmando que "la tasa de crecimiento de un país, relativa a la de otros países, puede ser aproximada por la relación entre la elasticidad ingreso de las exportaciones respecto a la elasticidad ingreso de las importaciones" (p. 66).

Zack y Dalle (2015), utilizando un modelo de corrección de error con datos trimestrales de importaciones y exportaciones, desde 1996 a 2013, miden la elasticidad precio e ingreso para las exportaciones e importaciones argentinas.

Albornoz (2018) utilizando un modelo de corrección de errores estudia las elasticidades del comercio exterior en diez países de América Latina, incluyendo a Ecuador, en el período 1993-2014, determinando 
la respuesta de las exportaciones e importaciones a cambios en el PIB y en los tipos de cambio. Por su parte Bergstrand, Egger y Larch (2013) analizaron la elasticidad sustitución.

En el caso de Ecuador, es importante nombrar el estudio publicado por el Banco Central del Ecuador de Gachet, Lastra, Loján, Ortiz, \& Pinzón (1998) como una primera aproximación al cálculo de la elasticidad de las importaciones por medio de técnicas de cointegración, en el cual se concluye que las importaciones en el período estudiado dependen en gran medida del PIB doméstico. También se han realizado trabajos a nivel de tesis de pregrado entre las que vale nombrar a (Quito, 2016) quien por medio de un estudio de series de tiempo estudió la elasticidad precio e ingreso para la demanda de las importaciones ecuatorianas en el período 2000-2015, llegando a conclusiones similares.

Chaney (2011) que analizó las elasticidades de las distancias y del tamaño de las economías y que afirmó que la elasticidad del comercio agregado con respecto al tamaño, eventualmente, disminuirá por debajo de uno para los países grandes.

Por medio de la aplicación del modelo gravitacional, en forma de un modelo econométrico de forma logarítmica, se puede llegar a determinar la elasticidad de los flujos comerciales bilaterales, ocasionada por cambios en las variables explicativas. Esto se evidencia en el trabajo de Montenegro, et al. (2011) sobre el efecto del crecimiento de la economía de China en sus importaciones desde América Latina, que utilizando un método de estimación de Poisson/binomial negativo (PML) determina los efectos del PIB chino como datos expresados en logaritmos y por lo tanto se muestra la elasticidad ingreso.

Pero, ¿cuáles son las bases para utilizar un modelo gravitacional? El modelo de gravedad o gravitacional aplicado al comercio exterior se basa en la ley de gravedad de Isaac Newton: la atracción entre dos cuerpos es proporcional al producto de sus masas y disminuye con la distancia existente entre ellos. Al colocar esta teoría en términos de comercio exterior se puede establecer que el volumen de comercio, entre dos países, depende del tamaño de sus economías y la distancia que los separa; es decir, la relación de intercambio bilateral es directamente proporcional al PIB de los dos países e inversamente proporcional a su distancia, todo esto en forma de un modelo econométrico simple.

Este modelo econométrico uniecuacional fue estudiado por primera vez por Timbergen (1962). Para el autor el término distancia se relaciona con los costos del comercio bilateral. Céterispáribus: a mayor distancia entre países, mayores costos y tiempo de transporte, y menor flujo de comercio; también incluye en este ámbito a las barreras legales al comercio. Pöyhönen (1963) aumentó variables al modelo para mejorar sus estimaciones entre las que se puede nombrar un coeficiente de la elasticidad de la renta nacional.

El modelo planteado tiene un carácter empírico, por lo que, en los años 70 se producen algunos estudios que introducen nuevos elementos al modelo, de esta manera, amplían su utilización al comercio de bienes diferenciados, en mercados de competencia monopolística y economías de escala crecientes. Los primeros fundamentos teóricos fueron dados por Anderson (1979), quien concluye que, la ecuación de gravedad puede derivarse de las propiedades de los sistemas de gasto.

En la década de 1980, investigaciones incorporan variables políticas, sociales y económicas al modelo. Bergstrand (1985) derivó la ecuación de gravedad a partir del equilibrio general del comercio mundial. Deardorff (1998) afirmó que el modelo se puede formular a partir de las teorías de comercio exterior como las de Ricardo e incluso mediante el modelo Heckscher-Ohlin.

Los autores de un documento de discusión de la Organización Mundial de Comercio afirman que el modelo tiene un alto valor explicativo de los flujos de comercio bilateral y que permite probar el papel que desempeñan otras variables que afectan al comercio (Piermartini \& Teh, 2005). Como afirman BacariaColom, Osorio-Caballero y Artal-Tur (2013) "la incorporación de los fundamentos teóricos a la práctica de la teoría de la gravedad ha dado lugar a una estimación más enriquecedora y más precisa de las relaciones espaciales que esta describe" (p. 152).

Según el Banco Mundial (2015), el modelo de gravedad es el de más común uso para la construcción de modelos de flujos comerciales entre países: "Este comenzó a aplicarse al principio como método empírico 
sensato que se adecuaba satisfactoriamente a los datos disponibles. Sin embargo, en tiempos más recientes se le ha dado una sólida base teórica" (p. 75) basada en las relaciones microeconómicas de consumidores y productores.

Appleyard y Field (2003) afirman que: "con frecuencia las pruebas empíricas que utilizan el modelo de gravedad han sido notablemente exitosas, lo cual significa que el volumen de comercio entre pares de países ha quedado bien explicado" (p. 175).

Krugman et al. (2012) describen la forma básica del modelo con la ecuación 1.

$$
T_{i j}=\frac{A \cdot Y_{i} \cdot Y_{j}}{D_{i j}}
$$

Dónde: $A$ es una constante, $T_{i j}$ es el valor del comercio entre el país $i$ y el país $j, Y_{i}$ es el PIB del país $i, Y_{j}$ es el PIB del país $j$ y $D_{i j}$ es la distancia entre los dos países (p. 13). En la constante $A$ están incluidas otras variables que influyen en los flujos comerciales.

El modelo utiliza a la distancia como variable proxy de los costos de comercio, Feenstra y Taylor (2011) sostienen que el comercio se ve influenciado por la cercanía entre los países, pues esta afecta a los costos de transporte. La distancia regularmente se mide entre las capitales de los países o entre las ciudades importantes para la industria y el comercio (centros gravitacionales) y el tamaño de la economía de cada país, como ya se especificó anteriormente, se mide en función de su PIB.

Según Egger y Pfaffermayr (2003), la aplicación del modelo gravitacional mediante el uso de datos de panel es muy común y puede ser desarrollada de varias formas. Los datos de panel son de mucha utilidad para analizar la misma unidad de corte transversal a lo largo del tiempo. Por esta característica y por la naturaleza misma de los datos necesarios para la aplicación del modelo de gravedad, es normal que se dé el uso de los datos de panel (Baltagi, Egger, \& Pfaffermayr, 2014).

De hecho, su uso es tan extendido que existen informes enteros sobre la aplicación de los datos de panel a los modelos de gravedad y las diferentes variaciones de este método en las que pueden ser aplicados, como es el caso de Baltagi, Egger, \& Pfaffermayr (2014). Se ha llegado al punto de que, como indica Bun y Klaassen (2002) "la mayoría de investigadores hoy en día usan datos de panel" al tratar el modelo de gravedad debido a que las series de tiempo adicionales permiten una mayor precisión en los estimados, a la vez que se evitan inconsistencias que presentan otros métodos de análisis.

Cuando se habla de los datos de panel, las formas más comunes de aplicarlos son la de efectos fijos y la de efectos aleatorios. Sin embargo, los efectos fijos no permiten la presencia de datos invariables durante todo el periodo de estudio, mientras que, los efectos aleatorios sí lo permiten. Así, la presencia de la distancia, no permite el análisis de los datos de panel mediante el método de efectos fijos (Carrère, 2006).

Estudios realzados sobre el Ecuador (Nagao, 2016)(Albornoz, 2019) y sobre países vecinos como Perú (Wang \& Badman, 2016) también han utilizado este método. En estos casos, los resultados han sido similares a los brindados por el método con efectos fijos.

Otras consideraciones que se tienen en cuenta en referencia al uso de datos de panel son las siguientes:

Primero, cuando los países tienen industrias más maduras, estas suelen desarrollar redes de mercadeo y cadenas de suministros, disminuyendo las barreras comerciales y, por lo tanto, afectando al comercio, lo que crea una relación entre el comercio presente y el de los años anteriores; sin embargo, esta afirmación no parece ser siempre importante (Bun \& Klaassen, 2002). Segundo, es normal que algunos países, especialmente cuando tienen economías relativamente pequeñas, tengan años en los que su comercio con alguno de sus socios comerciales sea cero, esto crea inconvenientes al realizar las estimaciones mediante datos de panel y por lo tanto pueden requerir un tratamiento diferenciado (Baltagi, Egger, \& Pfaffermayr, 2014). 


\section{Materiales y MÉtodos}

Para aplicar el modelo gravitacional, en el caso de Ecuador, en primera instancia se tomó como periodo de referencia 18 años, del 2000 al 2017, desde el momento en el cual el Ecuador fue dolarizado, un impacto que marca un antes y un después en la economía nacional, hasta el último año en el que está disponible toda la información. Se seleccionaron los 19 países con los cuales Ecuador tiene mayor comercio (más del 0,1\% del total del comercio con el continente) y cuyos datos de los intercambios comerciales no registren valores cero durante todo el periodo de análisis. Los países seleccionados son: Argentina, Bolivia, Brasil, Canadá, Chile, Colombia, Costa Rica, Cuba, El Salvador, Estados Unidos, Guatemala, Honduras, México, Nicaragua, Panamá, Paraguay, Perú, República Dominicana y Uruguay. Los países excluidos no tienen flujos comerciales que hayan sido considerados como relevantes para este documento, como se explicó anteriormente, y por lo tanto no es necesario utilizar una aproximación especial para tratar con los valores cero. El único país excluido y que se puede considerar que tiene un comercio relevante para el Ecuador es Venezuela; sin embargo, la situación actual de este país no permite obtener datos confiables sobre sus indicadores económicos, existiendo mucha discrepancia entre cifras oficiales y otras estimaciones, por lo que incluirlo podría afectar negativamente las estimaciones del modelo.

Los datos de importaciones y exportaciones fueron obtenidos de las dos bases de datos disponibles en el Banco Central del Ecuador (2019), la primera abarca el periodo comprendido entre los años 1990 y 2012 y la segunda desde el 2013 en adelante, en base al Incoterm free on board o libre abordo (FOB), los cuales estaban expresados en dólares estadounidenses. Una vez seleccionados los países, se obtuvieron los datos del PIB en valores nominales en dólares estadounidenses de la base de datos del Banco Mundial (2019).

El modelo propuesto asume que las variables del PIB de cada país y su distancia son independientes, y que los flujos comerciales, importaciones y exportaciones pueden explicarse en función de estas.

Se ha discutido mucho sobre si en la aplicación del modelo de gravedad se debe utilizar el PIB en términos reales o nominales. En el trabajo realizado por De Benedictis y Taglioni (2010) se afirma que es mejor utilizar esta variable en términos nominales puesto que, se utilizan índices de precios para eliminar la influencia de los precios en la contabilización de la producción de una economía, pero estos al ser calculados de formas distintas por los países no muestran los precios verdaderos a los que se comercializan los productos, por lo que, a la larga puede generar resultados sesgados en el modelo.

En este caso, se utilizan los valores nominales del PIB. Esta decisión se basa en algunos aspectos: primero, metodológicamente pueden existir problemas debido a la posibilidad de que los países reporten la información de los PIB reales con diferentes años base y segundo, no se podrían hacer estimaciones adecuadas teniendo mediciones de las importaciones y exportaciones en términos FOB a precios de mercado y comparándolas con los valores del PIB real.

La variable distancia está medida en kilómetros de manera ortodrómica, medida en línea recta entre dos puntos sobre una esfera, entre las capitales de los países para lo cual se utilizó la herramienta web Distance Calculator (2019).

Una vez obtenidos los datos, por la naturaleza de los mismos, estos son planteados como datos de panel. La importancia del uso de esta técnica básicamente se da por dos razones: a) heterogeneidad de los datos y b) la combinación de datos de series de tiempo, con datos de corte transversal, proporciona mayor cantidad de datos informativos, menos colinealidad y más grados de libertad y eficiencia, en concreto, cuando la misma unidad transversal (información de 19 países para un año determinado) se estudia a lo largo del tiempo (año 2000 al 2017), es decir, combina dos dimensiones, espacio y tiempo.

La especificación general de los modelos de regresión con datos de panel, planteados son las ecuaciones 2,3 y 4 . 


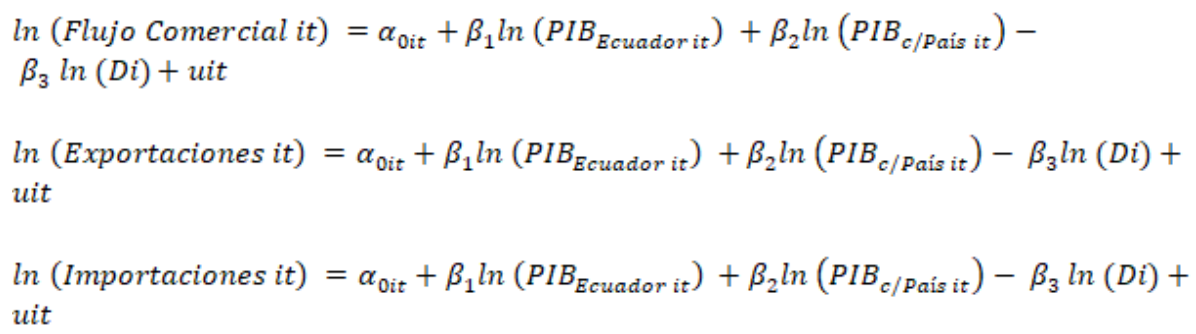

Donde:

$i=1,2,3,4,5 \ldots \ldots . \mathrm{N}$

$t=1,2,3,4,5 \ldots . . \mathrm{T}$

Donde $i$ se refiere a cada individuo o unidad de estudio (en nuestro caso los países), $t$ la dimensión de tiempo, $\alpha_{0 i t}$ el vector de interceptos de los $\mathrm{n}$ parámetros y $\beta$ es el vector de $k$ parámetros y es la $i$-ésima observación al momento $t$ para las $k$ variables explicativas. Por lo que el total de observaciones es $N x T$ (342 observaciones).

El término de error incluido en la regresión, se puede descomponer de la manera expresada en la ecuación 5.

$$
U_{i t}=\mu_{i}+\delta_{t}+\varepsilon_{i t}
$$

Donde:

$\mu_{\mathrm{i}}$ representa los efectos no observables que difieren entre las unidades de estudio (países), pero no en el tiempo.

$\delta_{\mathrm{r}}$ representa los efectos no cuantificables, que varían en el tiempo, pero no entre las unidades de estudio (países).

$\varepsilon_{\mathrm{it}}$ es el término de error aleatorio.

Si no existe heterogeneidad no observable entre los países, se satisfacen los supuestos del modelo lineal general, por lo que el método de MCO produce los mejores estimadores lineales insesgados.

Si suponemos a un efecto fijo y distinto para cada país, la heterogeneidad no observable se incorpora a la constante del modelo, por lo que supone un término constante para cada país y los efectos individuales son diferentes entre sí. En cambio, para efectos aleatorios, suponemos que los países, considerados en la muestra, tienen características similares, lo que supone que tienen una media común para el intercepto y que las diferencias individuales en los valores de la intercepción de cada país se reflejan en el término de error, es decir, considera que los efectos individuales no son independientes entre sí, sino que están aleatoriamente alrededor de un valor dado.

Así, en el caso específico de este artículo se utiliza el modelo de datos de panel con efectos aleatorios para poder comprender los efectos generados por la interacción comercial entre Ecuador y los diferentes países. Este método es elegido debido a la presencia de parámetros que no varían con el paso del tiempo, en este caso la distancia.

Se obtienen, a través del software EViews, resultados tanto para las importaciones y exportaciones, como para los flujos totales. 


\section{Resultados y DisCuSióN}

En todas las estimaciones las variables tienen el signo esperado, los tres modelos (flujos totales, exportaciones e importaciones) sugieren que el aumento en el PIB ecuatoriano, como en el PIB del resto de países, está asociado a aumentos en los flujos comerciales, exportaciones e importaciones (variables dependientes), mientras que la variable distancia tiene un efecto negativo en las variables antes mencionadas. Vale destacar que los coeficientes obtenidos describen de manera agregada a las elasticidades.

En la especificación del modelo se planteó rezagar la variable PIB. Sin embargo, en los tres modelos los rezagos no fueron estadísticamente significativos, obteniendo un p-value muy por arriba del 5\%. Adicionalmente, no se presentaron cambios significativos en la significancia global del modelo reflejado en el $R^{2}$.

En las Tablas 1,2 y 3 se pueden ver las estimaciones de los efectos fijos por país y por unidad de tiempo, si se desea conocer el efecto de las variables independientes sobre el flujo comercial, las exportaciones e importaciones entre el Ecuador y el resto de países a través de los efectos aleatorios.

TABLA 1.

\section{Resultado de efectos aleatorios al flujo total}

Cross-section Random
Effects
\begin{tabular}{lll}
\hline$\#$ & PAIS & Effect \\
\hline 1 & Argentina & 0,773197 \\
2 & Bolivia & $-0,538009$ \\
3 & Brasil & 0,188271 \\
4 & Canadá & $-0,508354$ \\
5 & Chile & 2,061361 \\
6 & Colombia & 0,412469 \\
7 & Costa Rica & $-1,444292$ \\
8 & Cuba & $-2,035302$ \\
9 & El Salvador & 0,119009 \\
& Estados & \\
10 & Unidos & 1,368454 \\
11 & Guatemala & $-0,126794$ \\
12 & Honduras & $-0,774421$ \\
13 & México & $-0,073833$ \\
14 & Nicaragua & $-0,457087$ \\
15 & Panamá & 1,683903 \\
16 & Paraguay & $-1,188515$ \\
17 & Perú & 1,255691 \\
& República & \\
18 & Dominicana & $-1,125039$ \\
19 & Uruguay & 0,409293
\end{tabular}

Los autores 
TABLA 2.

Resultado de efectos aleatorios a exportaciones

\begin{tabular}{|c|c|c|}
\hline \multicolumn{3}{|c|}{$\begin{array}{l}\text { Cross-section Random } \\
\text { Effects }\end{array}$} \\
\hline$\#$ & PAIS & Effect \\
\hline 1 & Argentina & 0,491598 \\
\hline 2 & Bolivia & $-0,369792$ \\
\hline 3 & Brasil & $-1,380613$ \\
\hline 4 & Canadá & $-0,780613$ \\
\hline 5 & Chile & 2,649844 \\
\hline 6 & Colombia & $-1,057281$ \\
\hline 7 & Costa Rica & $-1,956184$ \\
\hline 8 & Cuba & $-1,177214$ \\
\hline 9 & $\begin{array}{l}\text { El Salvador } \\
\text { Estados }\end{array}$ & 1,08817 \\
\hline 10 & Unidos & 1,420781 \\
\hline 11 & Guatemala & 0,615426 \\
\hline 12 & Honduras & 0,159613 \\
\hline 13 & México & $-1,202236$ \\
\hline 14 & Nicaragua & 0,476286 \\
\hline 15 & Panamá & 1,426302 \\
\hline 16 & Paraguay & $-1,422964$ \\
\hline 17 & $\begin{array}{l}\text { Perú } \\
\text { República }\end{array}$ & 1,257578 \\
\hline 18 & Dominicana & $-0,366891$ \\
\hline 19 & Uruguay & 0,128191 \\
\hline
\end{tabular}

Los autores

TABLA 4.

Resultado de efectos aleatorios a importaciones

\begin{tabular}{|c|c|c|}
\hline \multicolumn{3}{|c|}{$\begin{array}{l}\text { Cross-section Random } \\
\text { Effects }\end{array}$} \\
\hline$\#$ & PAIS & Effect \\
\hline 1 & Argentina & 1,261376 \\
\hline 2 & Bolivia & $-0,080586$ \\
\hline 3 & Brasil & 1,10048 \\
\hline 4 & Canadá & $-0,016563$ \\
\hline 5 & Chile & 1,979331 \\
\hline 6 & Colombia & 2,231368 \\
\hline 7 & Costa Rica & $-0,293798$ \\
\hline 8 & Cuba & $-2,845354$ \\
\hline 9 & $\begin{array}{l}\text { El Salvador } \\
\text { Estados }\end{array}$ & $-2,270436$ \\
\hline 10 & Unidos & 1,451243 \\
\hline 11 & Guatemala & $-0,716974$ \\
\hline 12 & Honduras & $-2,199633$ \\
\hline 13 & México & 0,891891 \\
\hline 14 & Nicaragua & $-3,026264$ \\
\hline 15 & Panamá & 2,615436 \\
\hline 16 & Paraguay & $-0,676758$ \\
\hline 17 & $\begin{array}{l}\text { Perú } \\
\text { República }\end{array}$ & 1,710218 \\
\hline 18 & Dominicana & $-1,911373$ \\
\hline 19 & Uruguay & 0,796395 \\
\hline
\end{tabular}

Los autores 
Vale la pena mencionar que los países con los que tenemos mayor flujo comercial, exportaciones o importaciones son: Colombia, Chile, Estados Unidos, Panamá y Perú, ya que sus efectos están arriba de la media.

Los resultados obtenidos con respecto a los flujos comerciales se muestran en el Gráfico l.

\begin{tabular}{|c|c|c|c|c|}
\hline \multicolumn{5}{|c|}{$\begin{array}{l}\text { Dependent Variable: LFC } \\
\text { Method: Panel EGLS (Cross-section random effects) } \\
\text { Date: } 06 / 29 / 19 \text { Time: } 14: 56 \\
\text { Sample: } 20002017 \\
\text { Periods included } 18 \\
\text { Cross-sections included: } 19 \\
\text { Total panel (balanced) observations: } 342 \\
\text { Swamy and Arora estimator of component variances }\end{array}$} \\
\hline Variable & Coefficient & Std. Error & t-Statistic & Prob. \\
\hline LPIBP & 0.643011 & 0.103068 & 6.238723 & 0.0000 \\
\hline LPIBE & 0.493102 & 0.096607 & 5.104208 & 0.0000 \\
\hline LD & -1.264378 & 0.486738 & -2.597658 & 0.0098 \\
\hline c & 2.219438 & 3.735720 & 0.594112 & 0.5528 \\
\hline \multicolumn{5}{|c|}{ Effects Specification } \\
\hline & & & S.D. & Rho \\
\hline Cross-section randon & & & 1.069599 & 0.8062 \\
\hline Idiosyncratic random & & & 0.524367 & 0.1938 \\
\hline \multicolumn{5}{|c|}{ Weighted Statistics } \\
\hline R-squared & 0.528896 & \multirow{5}{*}{\multicolumn{2}{|c|}{$\begin{array}{l}\text { Mean dependent var } \\
\text { S.D. dependent var } \\
\text { Sum squared resid } \\
\text { Durbin-Watson stat }\end{array}$}} & 0.592462 \\
\hline Adjusted R-squared & 0.524715 & & & 0.767771 \\
\hline S.E. of regression & 0.529309 & & & 94.69667 \\
\hline F-statistic & 126.4881 & & & 0.594493 \\
\hline Prob(F-statistic) & 0.000000 & & & \\
\hline \multicolumn{5}{|c|}{ Unweighted Statistics } \\
\hline R-squared & & \multirow{2}{*}{\multicolumn{2}{|c|}{$\begin{array}{l}\text { Mean dependent var } \\
\text { Durbin-Watson stat }\end{array}$}} & 5.161341 \\
\hline Sum squared resid & 492.4097 & & & 0.114329 \\
\hline
\end{tabular}

\section{Resultados del programa EViews referentes a los flujos totales}

Los autores.

Con los coeficientes obtenidos se plantea la ecuación 6 .

$$
\begin{aligned}
& \ln (\text { Flujo Comercial ti })=2,2194+0,4931 \ln \left(\text { PIB }_{\text {Ecuadorit }}\right)+ \\
& 0,6430 \ln \left(\text { PIB }_{\text {c/Pais it }}\right)-1,2643 \ln (D i)+\text { uit }
\end{aligned}
$$

\section{$\mathrm{F}: 126,48$}

\section{$R^{2}: 0,5288$}

Como se puede apreciar, las variables independientes son estadísticamente significativas y en conjunto explican en 52,88\% a los flujos comerciales del Ecuador con los países seleccionados.

La interpretación de los coeficientes es la siguiente:

Por cada $1 \%$ que aumente el PIB de Ecuador, Céteris páribus, los flujos comerciales aumentan en 0,4331\%. Por cada $1 \%$ que aumente el PIB de los otros países, Céteris páribus, los flujos comerciales aumentan en $0,6130 \%$. Al ser los valores menores a $1 \%$ los flujos comerciales son inelásticos al ingreso.

En el caso de la distancia por cada $1 \%$ que aumente, Céteris páribus, los flujos comerciales disminuyen en $1,2643 \%$.

En lo referente a las exportaciones, se pueden observar los resultados en el Gráfico 2. 


\begin{tabular}{|c|c|c|c|c|}
\hline \multicolumn{5}{|c|}{$\begin{array}{l}\text { Dependent Variable: LX } \\
\text { Method: Panel EGLS (Cross-section random effects) } \\
\text { Date: } 06 / 29 / 19 \text { Time: } 14: 58 \\
\text { Sample: } 20002017 \\
\text { Periods included: } 18 \\
\text { Cross-sections included: } 19 \\
\text { Total panel (balanced) observations: } 342 \\
\text { Swamy and Arora estimator of component variances }\end{array}$} \\
\hline Variable & Coefficient & Std. Error & t-Statistic & Prob. \\
\hline LPIBP & 0.834879 & 0.120314 & 6.939188 & 0.0000 \\
\hline LPIBE & 0.308015 & 0.111225 & 2.769299 & 0.0059 \\
\hline LD & -2.175952 & 0.593144 & -3.668503 & 0.0003 \\
\hline C & 8.203496 & 4.553062 & 1.801754 & 0.0725 \\
\hline \multicolumn{5}{|c|}{ Effects Specification } \\
\hline & & & S.D. & Rho \\
\hline Cross-section random & & & 1.313405 & 0.8349 \\
\hline Idiosyncratic random & & & 0.584162 & 0.1651 \\
\hline \multicolumn{5}{|c|}{ Weighted Statistics } \\
\hline R-squared & 0.479176 & \multirow{5}{*}{\multicolumn{2}{|c|}{$\begin{array}{l}\text { Mean dependent var } \\
\text { S.D. dependent var } \\
\text { Sum squared resid } \\
\text { Durbin-Watson stat }\end{array}$}} & 0.442294 \\
\hline Adjusted R-squared & 0.474553 & & & 0.805290 \\
\hline S.E. of regression & 0.583736 & & & 115.1728 \\
\hline F-statistic & 103.6572 & & & \\
\hline Prob(F-statistic) & 0.000000 & & & \\
\hline \multicolumn{5}{|c|}{ Unweighted Statistics } \\
\hline & 0.578268 & \multirow{2}{*}{\multicolumn{2}{|c|}{$\begin{array}{l}\text { Mean dependent var } \\
\text { Durbin-Watson stat }\end{array}$}} & 4.242153 \\
\hline Sum squared resid & 612.2782 & & & 0.151801 \\
\hline
\end{tabular}

GRÁFICO 2.

Resultados del programa EViews referentes a las exportaciones Los autores.

Con los coeficientes obtenidos se plantea la ecuación 7.

$$
\begin{aligned}
& \ln (\text { Exportaciones } t i)=8,2034+0,3080 \ln \ln \left(P I B_{\text {Ecuador it }}\right)+ \\
& 0,8348 \ln \left(P_{\text {c }}\right. \text { / Pais it } \\
& )-2,1759 \ln (D i)+\text { uit }
\end{aligned}
$$

\section{$\mathrm{F}: 103,65$}

\section{$R^{2}: 0,4791$}

Como se puede apreciar las variables independientes son estadísticamente significativas y en conjunto explican en $47,91 \%$ a las exportaciones del Ecuador a los países seleccionados.

La interpretación de los crecientes es la siguiente: Por cada 1\% que aumente el PIB de Ecuador, céteris páribus, sus exportaciones aumentan en $0,3080 \%$ esto muestra también la propensión a exportar. Por cada $1 \%$ que aumente el PIB de los otros países, céteris páribus, las exportaciones aumentan en $0,8348 \%$. $\mathrm{Al}$ ser los valores menores a $1 \%$ las exportaciones son inelásticas al ingreso.

En el caso de la distancia por cada $1 \%$ que aumente, céteris páribus, las exportaciones disminuyen en $2,1759 \%$.

En lo referente a las importaciones se muestran en el Gráfico 3. 


\begin{tabular}{|c|c|c|c|c|}
\hline \multicolumn{5}{|c|}{$\begin{array}{l}\text { Dependent Variable: LM } \\
\text { Method: Panel EGLS (Cross-section random effects) } \\
\text { Date: } 06 / 29 / 19 \text { Time: } 14: 59 \\
\text { Sample: } 20002017 \\
\text { Periods included: } 18 \\
\text { Cross-sections included: } 19 \\
\text { Total panel (balanced) observations: } 342 \\
\text { Swamy and Arora estimator of component variances }\end{array}$} \\
\hline Variable & Coefficient & Std. Error & t-Statistic & Prob. \\
\hline LPIBE & 0.971212 & 0.137476 & 7.064577 & 0.0000 \\
\hline LPIBP & 0.567432 & 0.146760 & 3.866388 & 0.0001 \\
\hline LD & -0.469217 & 0.694236 & -0.675876 & 0.4996 \\
\hline $\mathrm{C}$ & -9.521340 & 5.328265 & -1.786949 & 0.0748 \\
\hline \multicolumn{5}{|c|}{ Effects Specification } \\
\hline & & & S.D. & Rho \\
\hline Cross-section random & & & 1.526054 & \\
\hline Idiosyncratic random & & & 0.745160 & 0.1925 \\
\hline \multicolumn{5}{|c|}{ Weighted Statistics } \\
\hline & 0.506441 & \multirow{5}{*}{\multicolumn{2}{|c|}{$\begin{array}{l}\text { Mean dependent var } \\
\text { S.D. dependent var } \\
\text { Sum squared resid } \\
\text { Durbin-Watson stat }\end{array}$}} & \\
\hline Adjusted R-squared & 0.502060 & & & 1.080667 \\
\hline S.E. of regression & 0.762571 & & & 196.5521 \\
\hline F-statistic & 115.6073 & & & 1.002418 \\
\hline Prob(F-statistic) & 0.000000 & & & \\
\hline \multicolumn{5}{|c|}{ Unweighted Statistics } \\
\hline $\begin{array}{l}\text { R-squared } \\
\text { Sum squared resid }\end{array}$ & $\begin{array}{l}0.498045 \\
1235.525\end{array}$ & \multicolumn{2}{|c|}{$\begin{array}{l}\text { Mean dependent var } \\
\text { Durbin-Watson stat }\end{array}$} & $\begin{array}{l}3.955828 \\
0.159469\end{array}$ \\
\hline
\end{tabular}

\section{Resultados del programa EViews referentes a las exportaciones}

Los autores.

Con los coeficientes obtenidos se plantea la ecuación 8 .

$$
\begin{aligned}
& \ln (\text { Importaciones } t i)=-9,5213+0,9712 \ln \left(\text { PIB }_{\text {Ecuador it }}\right)+ \\
& 0,5674 \ln \left(\text { PIB }_{\text {c/Pais it }}\right)-0,4692 \ln (D i)+\text { uit }
\end{aligned}
$$

$\mathrm{F}: 115,60$

$R^{2}: 0,5064$

Como se puede apreciar, tanto las variables PIB Ecuador como las PIB de otros países son significativas, mientras que la distancia no y en conjunto explican en 50,64\% a las importaciones del Ecuador desde los países seleccionados.

La interpretación de los coeficientes es la siguiente:

Por cada $1 \%$ que aumente el PIB de Ecuador, céteris páribus, sus importaciones aumentan en 0,9012\%. Por cada $1 \%$ que aumente el PIB de los otros países, céteris páribus, las importaciones aumentan en 0,5674\%. $\mathrm{Al}$ ser los valores menores a $1 \%$ las exportaciones son inelásticas al ingreso.

En el caso de la distancia por cada $1 \%$ que aumente, céteris páribus, las importaciones disminuyen en $0,4692 \%$.

En el caso de Ecuador, estudios anteriores como el de Albornoz (2018) ya mostraron que el principal determinante de los volúmenes de comercio exterior es el PIB. Con mayor anterioridad, Gachet, Lastra, Loján, Ortiz y Pinzón (1998) ya indicaban que "las importaciones del Ecuador, tanto en el corto como en el largo plazo, son explicadas en gran medida por el ingreso nacional” (p. 118). El presente documento confirma estos hallazgos, al demostrar que el PIB es, nuevamente, el factor más significativo.

El modelo también indica que la elasticidad-ingreso de las importaciones es mayor a la elasticidad-ingreso de las exportaciones en el caso de Ecuador. Este resultado concuerda con el de (Albornoz, 2018), que indica en su estudio: 
"la elasticidad-ingreso de las importaciones es superior a la elasticidad-ingreso de las exportaciones en la mayoría de los países, por lo cual la región registraría una tendencia al deterioro de la balanza comercial bajo condiciones de crecimiento económico equilibrado" (p. 63).

Este resultado en particular tiene fuertes implicaciones para la discusión. Como indican Zack \& Dalle (2015):

"una economía que presente una elasticidad ingreso de las importaciones superior a la elasticidad ingreso de las exportaciones, céteris páribus, se va a ver obligada a crecer a un menor ritmo que sus socios comerciales, ya que en caso contrario incurrirá en un déficit comercial cada vez mayor" (p. 135).

Al largo plazo, esto también implica que si un país tiene una elasticidad-ingreso de las importaciones mayor a su elasticidad-ingreso de las exportaciones, experimentará un crecimiento mayor de sus importaciones y un deterioro en su balanza comercial (Thirlwall, 1979). Esto sería un lastre para el desarrollo de una economía como la ecuatoriana pues, ante un mayor crecimiento, habría una tendencia a importar cada vez más, traspasando los valiosos recursos económicos fuera del alcance de la industria nacional.

Los altos niveles de crecimiento de las importaciones y el reducido nivel de crecimiento de las exportaciones, ya han sido motivo de preocupación en el pasado. Esto, considerando especialmente, que en el caso del Ecuador, la situación es aún más compleja al tener una economía dolarizada por lo que el país es incapaz de devaluar su moneda (Vos \& León, 2003). Una economía de este tipo depende en gran medida del comercio internacional para mantener la liquidez y por lo tanto, se ve afectada de forma preocupante si sus importaciones son mayores que sus exportaciones, pues más dinero saldría de la economía y obviamente, la masa monetaria podría reducirse, afectando a la población al reducir su capacidad de consumo (Vos \& León, 2003). A largo plazo, esto afectaría a la balanza de pagos. Así, en el caso ecuatoriano, tener una elasticidad mayor en las importaciones que en las exportaciones podría implicar un riesgo mayor y que va más allá del lastre al desarrollo económico que ya ha sido descrito anteriormente, pues implica la posibilidad de futuras crisis si no se toman acciones que reduzcan este problema.

La posible explicación de la diferencia entre las elasticidades puede ser la misma que la descrita por Dutt Amitava (2002):

"A menudo se argumenta que los países pobres tienen en general, mayores elasticidades ingreso de la demanda por importaciones en comparación a los países ricos, porque aquellos producen bienes relativamente inelásticos, tales como bienes primarios y productos manufacturados básicos. Por lo tanto, a medida que el ingreso de los países pobres aumenta, estos demandan más bienes ingreso elásticos, y por lo tanto las importaciones se incrementan en mayor proporción al incremento del ingreso. Los países ricos no poseen esta característica, porque ellos son capaces de producir bienes relativamente sofisticados, ingreso elástico." (p. 79)

El Ecuador es un país que cumple con estas características. La mayoría de la producción del país es de materias primas o de bienes de bajo nivel de manufactura. Los productos más importantes para el Ecuador en los últimos años son los petroleros, seguidos de, sin un orden específico, el banano, el camarón, el atún, el cacao, el café, entre otros (BCE, 2019); demostrando que la mayor parte de la producción exportable son productos que cumplen con lo antes mencionado.

Las características de los bienes producidos en una economía, como lo concluía hace 40 años Thirlwall (1979), determinan tanto el atractivo de los bienes en el extranjero, como la propensión de un país a importar artículos de otros países. En otras palabras, debido a que en el Ecuador no hay artículos de un alto nivel de manufactura, como celulares, computadoras, medicinas o productos químicos; los ecuatorianos al aumentar su nivel de ingresos tienen que necesariamente recurrir al extranjero, condenando el desarrollo de la industria nacional en el proceso. Para empeorar la situación, los otros países no van a considerar mucho más atractivos a los productos ecuatorianos al mejorar sus economías. Esta es la razón por la cual el Ecuador puede caer en un círculo vicioso, en donde el crecimiento de la economía se vea limitado por su producción y esta, a su vez, 
sea incapaz de mejorar, debido a que no posee los ingresos necesarios, por no ser atractiva ni para sus propios ciudadanos, ni para los de otros países.

Como se mencionó en el párrafo anterior, los productos que importa el Ecuador son de un nivel de manufactura diferente al que exporta. Esto parece también indicar la razón del bajo coeficiente de la distancia en las importaciones. Los ecuatorianos al subir su ingreso buscarán productos con un mayor nivel de manufactura, productos que difícilmente podrían encontrar en el país y cuya sustitución es complicada.

Otra situación, aún más compleja es que existen productos, como las medicinas, que son indispensables para la población o para el funcionamiento de la industria local y que tampoco son producidos en el país. Por lo tanto, se entiende que, aunque suba la distancia, los ecuatorianos seguirán comprando una parte importante de estos productos en el extranjero, reduciendo la importancia real de la distancia.

Los coeficientes de elasticidad renta estimados, al estar expresados de manera agregada, deben reforzarse con otras herramientas para la toma de decisiones en cuanto a la política comercial del Ecuador. Por esto, se realizaron estimaciones por medio de $\mathrm{MCO}$ con respecto a las exportaciones del Ecuador y PIBs de sus socios comerciales, y las importaciones totales provenientes de cada país con respecto al PIB ecuatoriano.

Se eliminaron cinco países debido a que eran estadísticamente significativos o porque su $R^{2}$ era menor a 50\%. Los resultados se muestran en la Tabla 4.

TABLA 4.

Elasticidades ingreso por país

\begin{tabular}{|c|c|c|c|c|c|c|}
\hline \multicolumn{7}{|c|}{ Elasticidad Ingreso } \\
\hline & \multicolumn{3}{|c|}{ Exportaciones } & \multicolumn{3}{|c|}{ Importaciones } \\
\hline País & Coeficiente & R2 & Probabilidad & Coeficiente & R2 & Probabilidad \\
\hline Argentina & 1,2619 & 0,8886 & 0,000000005 & 0,9685 & 0,6486 & 0,000055057 \\
\hline Bolivia & 1,1313 & 0,9159 & 0,000000001 & 2,3477 & 0,6126 & 0,000123311 \\
\hline Brasil & 1,072 & 0,6063 & 0,000140899 & 0,9006 & 0,7449 & 0,000004005 \\
\hline Canadá & 1,74 & 0,7284 & 0,000006677 & 0,9828 & 0,9099 & 0,000000001 \\
\hline Chile & 2,2021 & 0,9121 & 0,000000001 & 0,4667 & 0,7462 & 0,000003842 \\
\hline Colombia & 0,7991 & 0,9002 & 0,000000002 & 0,6530 & 0,7541 & 0,000002972 \\
\hline Costa Rica & 0,9012 & 0,5710 & 0,000286855 & 1,0885 & 0,9176 & 0 \\
\hline Cuba & 1,1937 & 0,6633 & 0,000038804 & 2,2297 & 0,7667 & 0,000001938 \\
\hline $\begin{array}{l}\text { Estados } \\
\text { Unidos de }\end{array}$ & & & & & & \\
\hline América & 2,5942 & 0,6859 & 0,000021916 & 1,1330 & 0,8743 & 0,000000013 \\
\hline México & 2,1902 & 0,7580 & 0,000002612 & 1,2473 & 0,9069 & 0,000000001 \\
\hline Paraguay & 0,9275 & 0,5442 & 0,000475549 & 1,7032 & 0,6061 & 0,000141275 \\
\hline $\begin{array}{l}\text { Perú } \\
\text { República }\end{array}$ & 0,9327 & 0,6855 & 0,000022187 & 1,4876 & 0,8939 & 0,000000003 \\
\hline Dominicana & 1,2584 & 0,8303 & 0,000000146 & 2,6467 & 0,7869 & 0,000000928 \\
\hline Uruguay & 2,0215 & 0,8808 & 0,000000008 & 0,6056 & 0,5200 & 0,000732890 \\
\hline
\end{tabular}

Los autores.

Los países con coeficiente de elasticidad renta de las exportaciones, mayor a la unidad, en orden descendente, son: Estados Unidos de América, Chile, México, Uruguay, Canadá, Argentina, República Dominicana, Cuba, Bolivia y Brasil. En conjunto estos socios comerciales representan el 48,83\% del total de exportaciones del Ecuador y deben ser tomados muy en cuenta para los esfuerzos de potencialización de las exportaciones.

Los países con coeficientes de elasticidad renta de las importaciones, mayor a la unidad, en orden descendente, son: República Dominicana, Bolivia, Cuba, Paraguay, Perú, México, Estados Unidos de América, Costa Rica. En conjunto estos socios comerciales representan el 31,21\% del total de exportaciones del Ecuador. Se debe analizar detenidamente el tipo de productos que se importan de cada uno de estos países y determinar que potencialidad tiene Ecuador para sustituir las importaciones y tratar en algunos casos de tomar otro tipo de medidas que desalienten estas importaciones. 


\section{Conclusiones}

Los resultados demuestran que en los 19 países estudiados los flujos comerciales bilaterales, las exportaciones e importaciones, en el período analizado, dependen en gran medida del PIB de los países; se encuentran dentro de estos, a los tres socios comerciales más importantes del Ecuador en el continente americano (Estados Unidos de América, Colombia y Perú). Los 19 países representan en promedio, durante el periodo de estudio, el 63,46\% del comercio de Ecuador con el mundo.

Dada la importancia de los países del continente americano como destino de las exportaciones ecuatorianas, la política comercial debe estar encaminada a fomentar las exportaciones, por lo que, ProEcuador debe cumplir un papel fundamental realizando ruedas de negocios, misiones empresariales y demás estrategias de promoción. Además, en coordinación con el Ministerio de Relaciones Exteriores y Movilidad Humana, y el Ministerio de Producción Comercio Exterior Inversiones y Pesca se deben potencializar las oficinas comerciales ya existentes y fundar nuevas, sobre todo, en los países en los que el coeficiente de elasticidad ingreso sea mayor a la unidad. Sin descuidar el mercado de Estados Unidos de América se debe prestar especial atención a países como Chile, México, Uruguay, Canadá, Argentina y Brasil.

Se debe también analizar, con detenimiento, la situación de los socios comerciales más cercanos geográficamente, Colombia y Perú, pues para fomentar las exportaciones con estos países se debe tener muy en cuanta la complementariedad de la oferta exportable con respecto a su producción local.

Hay que prestar especial atención a la relación comercial con Perú, esto se debe a tener una mayor elasticidad renta en las importaciones que en las exportaciones y al alto flujo comercial que Ecuador tiene con ese país. Como se explicó anteriormente, tener una elasticidad renta de este tipo implica que Ecuador puede tener dificultades para crecer al mismo ritmo que Perú, por el lastre que generaría en la economía ecuatoriana.

Por otro lado, al tener Ecuador una economía dolarizada y no tener la posibilidad de tomar decisiones de política monetaria, como devaluación de la moneda, se debe continuar con la política de fomento a la sustitución de importaciones y en casos muy extremos, tomar medidas de carácter arancelarias y no arancelarias que podrían conllevar represalias comerciales con algunos de los países afectados.

Los valores y signos de los coeficientes de elasticidad-renta deberían ser considerados por los funcionarios gubernamentales, pues de esta manera, se puede orientar la política comercial, ya que estos funcionan como una importante herramienta para la toma de decisiones. Así, se puede utilizar la información disponible para analizar el comportamiento de los flujos comerciales en base a los pronósticos económicos y poder investigar la razón del cumplimiento o falta de cumplimiento de las expectativas teóricas. En ese aspecto, esta investigación es un primer paso para la formulación de las futuras políticas públicas y la generación de más investigación.

Este último punto debe ser considerado por la política económica ejecutada por el gobierno ecuatoriano, no tenerlo en cuenta implica que el Ecuador nunca podrá alcanzar un alto nivel de desarrollo o uno comparable a los países norteamericanos o de Europa Occidental, pues mientras más se acerque al nivel de desarrollo económico de estos, más difícil le será continuar ese acercamiento pues al crecer su economía, el lastre será mayor. Esto implica también un análisis de la producción ecuatoriana y la necesidad de buscar formas prácticas de generar su crecimiento, tanto para que los productores locales puedan sustituir, en medida de lo posible, las importaciones, como para que las características de la producción ecuatoriana sean más deseadas en el exterior. La sustitución de las importaciones es complicada ya que algunos productos difícilmente podrán ser remplazados, incluso en el largo plazo, y tampoco es posible hacerlo con todos los productos. 
Luis B. Tonon Ordóñez, et al. Elasticidad-renta del comercio bilateral mediante el Modelo Gravitac...

\section{REFERENCIAS BIBLIOGRÁFICAS}

Albornoz, A. C. (2019). Aplicación del Modelo de Gravedad entre Ecuador y la Unión Europea para el periodo 2001 2017. Cuenca. Obtenido de http://dspace.uazuay.edu.ec/handle/datos/8849

Albornoz, M. (2018). Elasticidades del comercio exterior en América Latina. Estimaciones para 1993 - 201. Ciclos(50), 61-86.

Andrerson, J. (1979). A Theoretical Foundation for Gravity Equation. American Economic Review, 69(1), 106-116.

Appleyard, D., \& Field, A. (2003). Economía internacional (4 ed.). McGraw-Hill.

Baltagi, B., Egger, P., \& Pfaffermayr, M. (2014). Panel Data Gravity Models of International Trade. En B. Baltagi, The Oxford Handbook of Panel Data (págs. 608-642). Nueva York: Oxford University Press.

Bacaria-Colom, J., Osorio-Caballero, M., \& Artal-Tur, A. (2013). Evaluación del Acuerdo de Libre Comercio MéxicoUnión Europea mediante un modelo gravitacional. Economia Mexicana, Nueva Epoca, 22(3), 143-163.

Banco Central del Ecuador. (2019). Banco Central del Ecuador.

Banco Mundial. (2015). ¿Cómo y por qué evolucionan los costos del comercio? En Banco Mundial, La ayuda para el comercio en sintesis 2015: Reducir los costos del comercio con miras a un crecimiento inclusivo y sostenible (págs. 69-92). Paris: Organisation for Economic Co-operation and Development / World Trade Organization.

Banco Mundial. (2019). World Bank Open Data. Recuperado el 25 de mayo de 2019

Bergstrand, J. (1985). The Gravity Equation in International Trade: Some Microeconomic Foundations and Empirical Evidence. The Review of Economics and Statistics, 67(3), 474-481.

Bergstrand, J., Egger, P., \& Larch, M. (2013). Gravity Redux: Estimation of gravity-equation coefficients, elasticities of substitution, and general equilibrium comparative statics under asymmetric bilateral trade costs. Journal of International Economics, 89(1), 110-121.

Bun, M., \& Klaassen, F. (2002). The importance of dynamics in panel gravity models of trade. En D. o. Economics, UvA Econometrics Discussion Paper. Amsterdam.

Carrère, C. (2006). Revisiting the effects of regional trade agreements on trade flows with proper specification of the gravity model. European Economic Review(50), 223-247.

Chaney, T. (2011). The Gravity Equation in International Trade. Review of Economics and Statistics, 475-481.

Daniels, J., Radebaugh, L., y Sullivan, D. (2013). Negocios internacionales (14 ed.). México: Pearson Edicación, S.A.

De Benedictis, L., \& Taglioni, D. (2013). The Gravity Model in International Trade. En L. de Benedictis, \& L. Salvatici (Edits.), The Trade Impact of European Union Preferential Policies: An Analysis Through Gravity Models (págs. 55-90). Springer.

Deardorff, A. (1998). Determinants of Bilateral Trade: Does Gravity Work in a Neoclassical World? NBER Working Paper No. W5377(January), 7-32.

Distance Calculator. (2019). Distance Calculator FInd Distance Between Cities. Recuperado el 20 de junio de 2019, de https://www.distancecalculator.net/

Dutt, K. (2002). La elasticidad ingreso de las importaciones, comercio Norte-Sur y desarrollo desigual. Cuestiones económicas(18), 59-89.

Egger, P., \& Pfaffermayr, M. (junio de 2003). The proper panel econometric specification of the gravity equation: A three-way model with bilateral interaction effects. Empirical Economics, 571-580.

Feenstra, R., \& Taylor, A. (2011). Comercio internacional (1 ed.). Barcelona: Reverté-Aguilar, S.L. Propiedad.

Gachet, I., Lastra, A., Loján, V., Ortiz, M., \& Pinzón, C. (1998). Cálculo de las elasticidades de la demanda total de importaciones en el Ecuador. En I. Gachet, A. Lastra, V. Loján, M. Ortiz, \& C. Pinzón. Quito: Banco Central del Ecuador.

Harberger, A. (1957). Some evidence on the international price mechanism. The Review of Economics and Statistics, 40(1), 123-127. 
Krugman, P., Obstfeld, M., \& Melitz, M. (2012). Economía international: Teoría y politica (9 ed.). Madrid: Pearson Edicación, S.A.

Marshall, A. (1890). Principios de Economia (Octava ed.). Prometheus.

Montenegro, C., Pereira, M., \& Soloaga, I. (2011). El efecto de China en el comercio internacional de América Latina. Estudios de Economia (38), 341-368.

Nagao, K. (2016). Estructura y Determinantes Principales del Comercio Internacional para el Ecuador. Quito.

Orcutt, G. (1950). Measurement of Price Elasticities in International Trade. The Review of Economics and Statistics, 32(2), 117-132.

Piermartini, R., \& Teh, R. (2005). Demystifying modelling methods for trade policy. World Trade Organization.

Pöyhönen, P. (1963). A Tentative Model for the Volume of Trade between Countries. Weltwirtschaftliches Archiv, 90(1963), 93-100.

Thirlwall, A. (1979). The Balance of Payments Constraint as an Explanation of International Growth Rate Differences. Banca Nazionale del Lavoro Quarterly Review, 128, 45-53.

Timbergen, J. (1962). Shaping the World Economy; Suggestions for an International Economic Policy. New York: Twentieth Century Fund.

Vos, R., \& León, M. (2003). Dolarización, dinámica de exportaciones y equidad: ¿cómo compatibilizarlas en el caso de Ecuador?

Wang, X., \& Badman, R. (2016). A Multifaceted Panel Data Gravity Model Analysis of Peru's Foreign Trade.

Yotov, Y., Larch, M., Monteiro, J.-A., \& Piermartini, R. (2016). An advanced guide to trade policy analysis: The structural gravity model. Geneva, Switzerland: World Trade Organization.

Zack, G., \& Dalle, D. (2014). Elasticidades del comercio exterior de la Argentina:¿ una limitación para el crecimiento? Revista Argentina de Economía Internacional, 3, 31-46.

(C) Universidad de Cuenca 2019

CC BY-NC-SA

\section{INFORMACIÓN ADICIONAL}

Código JEL: : F14 